\title{
Homa-IR: A Marker of Vascular Dysfunction in Nondiabetic Hemodialysis Patients?
}

\author{
Paraskevi Tseke Kimon Stamatelopoulos George Rammos Eirini Grapsa \\ Christos Papamichael Nikolaos Zakopoulos
}

Renal Unit, Vascular Laboratory, and Department of Clinical Therapeutics, General Hospital 'Alexandra',

University of Athens, Athens, Greece

We found the paper of Zhou et al. [1], recently published in your journal, of special interest. We would like to report that we observed similar findings in a group of 63 (27 female and 36 male) nondiabetic chronic hemodialysis patients.

Our patients, aged $60.5 \pm 12.1$ years, were on chronic hemodialysis for at least 6 months (median 38 months). Causes of renal disease were chronic glomerulonephritis (34.3\%), primary hypertension (27.1\%), polycystic kidney disease (7.1\%), chronic interstitial nephritis (7.2\%) and unknown (24.3\%). None of the patients was diagnosed to suffer from diabetes mellitus (based on medical history, or abnormal blood glucose). All patients were dialyzed on a 4-hour, thrice weekly schedule with a bicarbonate-buffered dialysate. All patients had a functioning vascular access (arteriovenous fistula or graft) and were clinically stable (no acute inflammatory event or cardiovascular event for the last 3 months, no malignant or severe hepatic disease). Twenty patients had a history of smoking, but none was a current smoker. Seventeen patients had a medical history remarkable for cardiovascular disease. All participants gave their informed consent to the study and the institute's ethics committee approved the study protocol.

Predialysis blood samples were taken on a midweek session to assess biochemical parameters, including glucose, insulin, CRP, total and HDL cholesterol, triglycerides, and apolipoproteins A and B. We estimated HOMA-IR (Homeostasis Model Assessment for Insulin Resistance) according to the formula: fasting glucose $(\mathrm{mmol} / \mathrm{l}) \times$ fasting insulin $(\mathrm{IU} / \mathrm{ml}) / 22.5$ [2]. All patients underwent B-mode ultrasonography of common carotid arteries (bilaterally) to obtain measures of arterial stiffness (including distensibility, compliance and carotid incremental elastic modulus; Einc index), and central (aortic) blood pressure was estimated using SphygmoCor, as we have previously described [3, 4]. Einc is an index of the elastic properties of vessels, which is inversely correlated to distensibility. Einc is calculated by the following mathematic formula: Einc = $3(1+$ LCSA/WCSA $) /$ distensibility. LCSA (lumen cross-sectional area) is estimated by the internal common carotid artery diameter $(\mathrm{CCI})$ as: $\mathrm{LCSA}=\pi(\mathrm{CCI} / 2)^{2}$, where $\pi=3.14$ and WCSA (wall cross-sectional area) is estimated by the external com- mon carotid artery diameter (CCE) as: $\pi(\mathrm{CCE} / 2)^{2}-\pi(\mathrm{CCI} / 2)^{2}$. Our patients had a median HOMA-IR value of 1.45 (range 0.39-5.26). In univariate regression analysis (using HOMA-IR in log scale as the dependent variable), we found HOMA-IR to be positively correlated with apolipoprotein $\mathrm{B}(\mathrm{b}=0.68, \mathrm{p}=0.008)$, LDL cholesterol $(b=0.006, p=0.001)$, non-HDL cholesterol (defined as total minus HDL cholesterol; $\mathrm{b}=0.006, \mathrm{p}<0.001)$, total cholesterol $(\mathrm{b}=0.005, \mathrm{p}=0.001), \mathrm{CRP}$ (in $\log$ scale; $\mathrm{b}=0.11, \mathrm{p}=0.05)$ and negatively with the apolipoprotein A/apolipoprotein $B$ ratio $(b=-0.21, p=0.016)$. In stepwise multivariate regression analysis, only CRP $(\mathrm{b}=0.13, \mathrm{p}=0.012)$ and non-HDL cholesterol $(b=0.005, p<0.001)$ were independently associated with HOMA-IR. There was no statistically significant association between HOMA-IR and intima-media thickness or plaque score $(p>0.05)$. Furthermore, in accordance to Zhou et al. [1], there was no significant association between HOMA-IR and cardiovascular disease $(p<0.05)$. In ordinal regression analysis using tertiles of HOMA-IR as the dependent variable, only Einc was associated with statistically significant odds for in-

\section{KARGER}

Fax +4161306 1234 E-Mail karger@karger.ch www.karger.com
Dr. Paraskevi Tseke

Renal Unit, General Hospital 'Alexandra'

Alexandroupoleos 48

GR-115 27 Athens (Greece)

Tel. +30 2107288 606, Fax +30 2107018 114, E-Mail tskedaki@hotmail.com 
creased HOMA-IR (odds ratio: 3.74, $\mathrm{p}=$ 0.017).

Although our findings regarding associations of HOMA-IR with dyslipidemia and arterial stiffness are in accordance to Zhou et al. [1], we noted that HOMA-IR was not correlated to cardiovascular disease itself or robust markers of advanced atherosclerosis (i.e. intima-media thickness, plaque score). It seems possible that HOMA-IR is a marker of early functional alterations rather than advanced structur- al abnormalities noted in vascular disease. However, to confirm any causal relationship, randomized longitudinal studies should be carried out.
References
1 Zhou Y, Yu Z, Jia H, et al: Association between insulin resistance and carotid arterial stiffness in nondiabetic hemodialysis patients. Blood Purif 2009;28:193-199.

-2 Matthews DR, Hosker JP, Rudenski AS, et al: Homeostasis model assessment: insulin resistance and beta-cell function from fasting plasma glucose and insulin concentrations in man. Diabetologia 1985;28:412-419.
3 Tseke P, Grapsa E, Stamatelopoulos K, et al: Correlations of sialic acid with markers of inflammation, atherosclerosis and cardiovascular events in hemodialysis patients. Blood Purif 2008;26:261-266.

4 Tseke P, Grapsa E, Stamatelopoulos K, et al: Atherosclerotic risk factors and carotid stiffness in elderly asymptomatic HD patients. Int Urol Nephrol 2006;38:801-809.
Tseke/Stamatelopoulos/Rammos/Grapsa/ Papamichael/Zakopoulos 